as barriers to hand contamination with gram-negative organisms and enterococci during hospital procedures, the researchers studied 137 procedures during which a healthcare worker's gloved hand contacted a patient's mucous membrane. The procedures included oral dental exams, endotracheal tube care, and digital rectal stimulation for bowel training. Quantitative hand cultures were obtained from each healthcare worker before and after the gloved contact.

The findings indicated that 86 of the 135 glove cultures had gram-negative rods or enterococci on the external surface after use and thus were sources of potential hand contamination. Microbial contamination of the healthcare worker's hands occurred in $\mathbf{1 1}$ (13\%) of those 86 events.

Contamination was more frequent with vinyl gloves, occurring in 10 of 42 cases studied compared with only one out of 44 cases involving latex gloves. After use, glove leaks also were more frequent in vinyl gloves (26 out of 61) than with latex gloves (6 of 70). Even when leaks were present, gloves prevented hand contamination in $77 \%$ of instances, and quantitative counts of microorganisms contaminating hands were 2 to $4 \operatorname{logs}$ less than counts on external surface of gloves. Healthcare workers reported awareness of the presence of glove leaks in only seven (22\%) of the 32 events in which leaks were subsequently demonstrated.

The authors conclude that gloves are an effective barrier to hand contamination and that the data confirm the need for handwashing routinely following removal of gloves.

FROM: Olsen RJ, et al. Examination gloves as barriers to hand contamination in clinical practice. JAMA 1993;270:350-353.

\section{Poor Antibody Response to Recombinant Hepatitis B Vaccines}

Following immunization with hepatitis B vaccine (Recombivax HB or Engerix-B) of 595 healthcare workers in 10 acute care hospitals in Minnesota, 11\% were found to be seronegative for antibody to hepatitis B surface antigen (anti-HBs). Postvaccination antibody testing was done within 6 months after receiving the third dose of vaccine. No deficiencies were identified in vaccine shipping or storage practices and all vaccines were administered in the deltoid muscle. Although different needle lengths were used, needle length was not associated with lack of anti-HBs. Dr. Rachel Wood and colleagues at the Minnesota Department of Health reported that five variables were found by multivariate analysis to be independently associated with lacking anti-HBs: vaccine brand, smoking status, gender, age, and body mass index.
Stratifying by vaccine brand demonstrated that age, body mass index, and smoking status were associated with lacking anti-HBs only for Engerix-B recipients. After controlling for smoking status, age, gender, and body mass index, recipients of Recombivax $\mathrm{HB}$ were more likely to lack anti-HBs than recipients of Engerix-B (relative risk, 2.3; 95\% confidence interval, 1.1 to $4.7 ; \mathbf{P}=\mathbf{0 . 0 2}$ ).

FROM: Wood RC, et al. Risk factors for lack of detectable antibody following hepatitis B vaccination of Minnesota healthcare workers. JAMA 1993;270:29352939.

\section{National HIV Information Network Launched}

A national computer-based information network has been launched to help healthcare providers track trends and treatment patterns for AIDS and HIV The CDC has awarded a 3-year, $\$ 900,000$ contract to suburban Chicago-based Dun \& Bradstreet Corp for the effort, intended to help direct healthcare providers in their care of HIV and AIDS patients.

Believed to be the first national effort of this type in the United States, the network, known as Health Research Network, will provide two types of information. It will follow individual but anonymous patients over the course of their illnesses, showing the effects of treatment approaches. The in-depth longitudinal data base also will allow the CDC to analyze trends and provide statistical overviews of the disease and its treatment.

The networks first effort involves a study of between 5,000 and 10,000 HIV-infected individuals whose care is being directed by infectious disease physicians in Atlanta, Los Angeles, San Francisco, Tampa, Florida, and Portland. Additional sites are expected to be added during the 3-year life of the grant.

CDC officials said that the system will be able to track the spectrum of HIV disease, including symp toms and use of medical therapy, drugs, and costs, information that is important to healthcare delivery. Hospitals, through participating physicians, will be able to tap into the network via computer to decide what services they may need to provide to care for patients with the disease.

FROM: Modem Health Care December 13, 1993.

\section{Shigella Strains Resistant to Fluoroquinolones}

The first fluoroquinolone-resistant Shigella 